\title{
Jets and QCD in photon-proton reactions
}

\section{Sascha Caron* (on behalf of the $\mathbf{H} \mathbf{1}$ and ZEUS collaborations)}

1. Phys. Institut, RWTH Aachen, Germany

E-mail: iscaron@mail. desy.de'

ABSTRACT: Recent single and dijet data from the HERA collider which give information on the scattering of real and virtual photons with protons are reviewed. These data provide additional information on the structure of the photon and the proton.

HERA has the potential to study photoproduction reactions at high transverse energy $E_{T}$. These reactions are investigated in inelastic electron (positron) proton reactions at very small squared four-momentum transfers $Q^{2}$.

A slightly off-shell photon has a probability to fluctuate into a quark pair before it enters the hard scattering. The longitudinal momentum fraction of the photon taken by the parton $x_{\gamma}$ is thus less than 1 (resolved), whereas interactions where the whole photon enters the hard scattering are given by $x_{\gamma} \approx$ 1 (direct). The resolved case is calculated using photon parton densities (pdf's) which are partly calculable in perturbative QCD. Jet cross sections measured in photoproduction allow a study of real photon structure, whereas at higher $Q^{2}$, virtual photon structure can be tested. The pdf's for virtual photons are calculable in perturbative QCD for the phase space $\Lambda^{2}<<Q^{2}<<E_{T}^{2}$. Note that the distinction between direct and resolved is

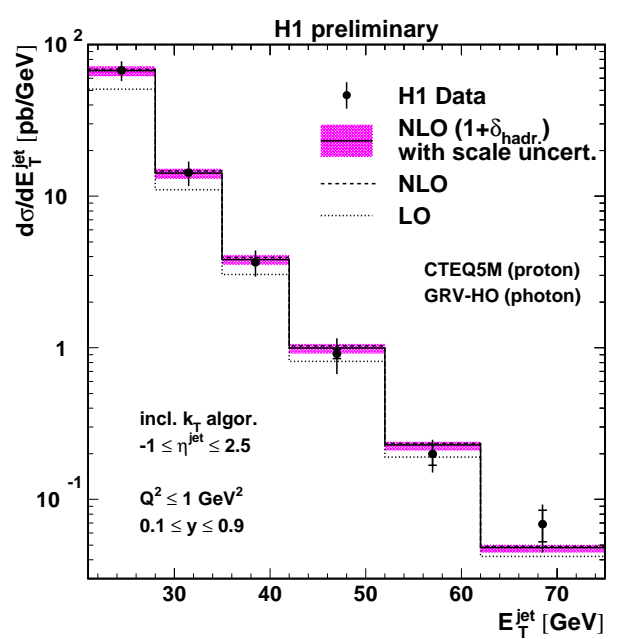

Figure 1: Differential ep cross section $d \sigma / d E_{T}^{J e t}$ for inclusive jet production. only unambiguously defined in leading order $(\mathrm{LO})$. In the presence of a hard scale $\left(E_{T}, Q^{2}\right)$ the jet cross sections can be calculated according to the factorization theorem of QCD as a convolution of the partonic cross sections of pointlike particles with parton momentum distributions of the proton and the photon. The partonic cross sections can be expanded as a perturbative series in powers of $\alpha_{s}$. Calculations up to next-to-leading order (NLO) are available [iin].

${ }^{*}$ Speaker. 
This article summarizes new measurements on jet production at very high $E_{T}$ in photoproduction $\left(Q^{2}<1 \mathrm{GeV}^{2}\right)$ and of moderate $E_{T}$ from low, but not vanishing $Q^{2}$ up to high $Q^{2}\left(0.1 \mathrm{GeV}^{2}<Q^{2}<10^{4} \mathrm{GeV}^{2}\right)$. The data's relevance is to test perturbative QCD including photon and proton parton densities. The data can also be important as a possible background to unexpected physics signals. All analyses discussed in the following use the

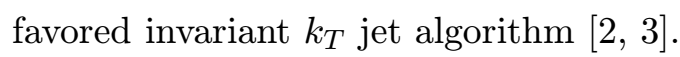

The H1 experiment has measured inclusive jet cross sections at high transverse energies $\left(E_{T}^{J e t}>21 \mathrm{GeV}\right)$ in photoproduction [i]. The inclusive differential cross section $d \sigma / d E_{T}^{J e t}$ as a function of $E_{T}^{\text {Jet }}$ is presented in fig. 1 for pseudorapidities $-1<\eta_{\text {Jet }}<2.5$

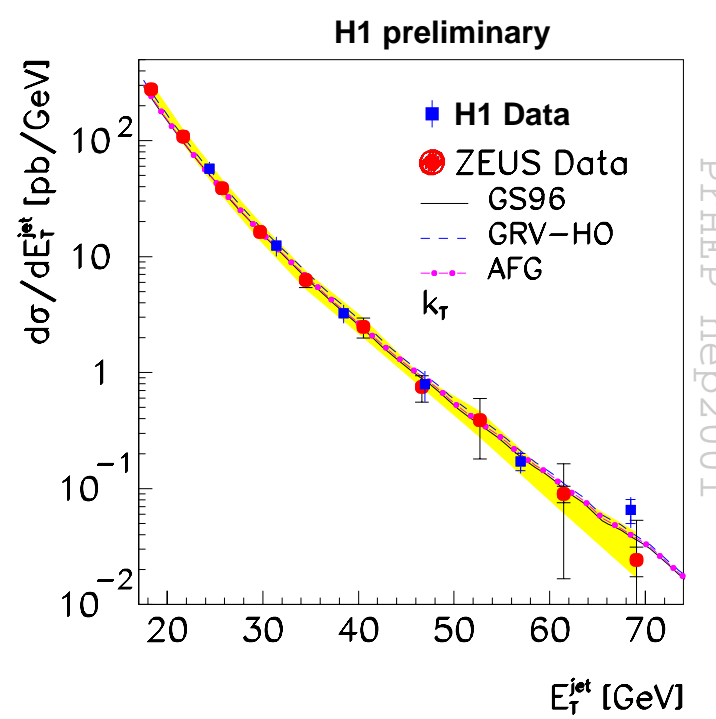
and inelasticities $0.1<y<0.9$. The inelasticity $y$ can here be interpreted as the fraction of the electron's longitudinal momentum taken by the photon. The data are compared with LO (dotted line) and NLO (dashed line) QCD calculations using the CTEQ5M parametrization of the proton pdf and the GRV-HO parametrization of the photon pdf.

Hadronization effects were found to be small $(<5 \%)$ and were included in the NLO expectation (full line). The renormalization scale and the factorization scales were set to the sum of the transverse energies of the outgoing partons divided by two. This common scale was varied from 0.5 to 2 times the default scale to estimate the scale uncertainty in the NLO calculation (grey band).

NLO QCD describes the data well up to $E_{T, \text { Jet }}$ of $70 \mathrm{GeV}$. Since there is only a $5-10 \%$ difference of the calculated NLO cross sections between using AFG-HO, GS-HO and GRV-HO photon pdf's, these pdf's would also describe the data. Fig. 2 shows the inclusive jet cross section $d \sigma / d E_{T}^{\text {Jet }}$ as a function of $E_{T}^{J e t}$ for a reduced $\eta_{J e t}$ and

Figure 2: Differential ep cross section $d \sigma / d E_{T}^{\text {Jet }}$ for inclusive jet production. $y$ range in order to compare the recent $\mathrm{H} 1$ result with a preliminary ZEUS measurement . The two measurements agree well within the uncertainties. 
Both experiments have also measured dijet cross sections, which make it possible to partly reconstruct the scaled longitudinal momenta $x_{\gamma}$ and $x_{P}$, from the two hardest jets produced in the hard subprocess

$$
\begin{aligned}
& x_{\gamma}=\frac{1}{2 E_{e} y}\left(E_{T, 1} e^{-\eta_{1}}+E_{T, 2} e^{-\eta_{2}}\right) \\
& x_{P}=\frac{1}{2 E_{P}}\left(E_{T, 1} e^{\eta_{1}}+E_{T, 2} e^{\eta_{2}}\right)
\end{aligned}
$$

$x_{P}$ is the momentum fraction of the proton taken by the hard scattering parton and $E_{e}$ and $E_{P}$ are the energies of the electron and proton beams.

These relations are exact in LO and are used as observables in all orders. The dijet cross section $d \sigma / d x_{\gamma}$ as a function of $x_{\gamma}$ measured by ZEUS is pictured in fig. 3 [i, $\left.\mathbf{6}^{-}\right]$. A $E_{T, 1}>$ $14 \mathrm{GeV}$ for the highest $E_{T}$ jet and $E_{T, 2}>11 \mathrm{GeV}$ for the second highest $E_{T}$ jet have been required. These cross sections are measured for four different regions in $E_{T, 1}$ and are compared with a NLO calculation using AFG-HO photon and CTEQ4M Proton pdfs.

Agreement between the data and the calculation is only observed for $x_{\gamma}>0.8$. The higher the cut in $E_{T, 1}$ the more the calculation underestimates the data for $x_{\gamma}<0.8$ (up to $60 \%$ ). However, this discrepancy is found to be larger than the uncertainties of the measurement and the estimation of the scale uncertainties in the NLO calculation. The H1 collaboration has also recently measured this cross section [i, $\left.\bar{i}_{-1}\right]$ as displayed in fig. 4 . In this analysis the jet selection criteria required $E_{T, 1}>$ $25 \mathrm{GeV}$ and $E_{T, 2}>15 \mathrm{GeV}$. The data are compared to NLO calculations using CTEQ5M proton and

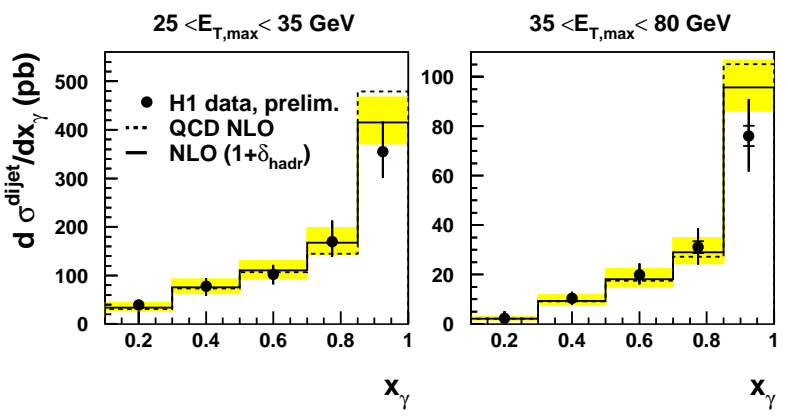

Figure 4: Differential ep cross section $d \sigma / d x_{\gamma}$ for dijet jet production.

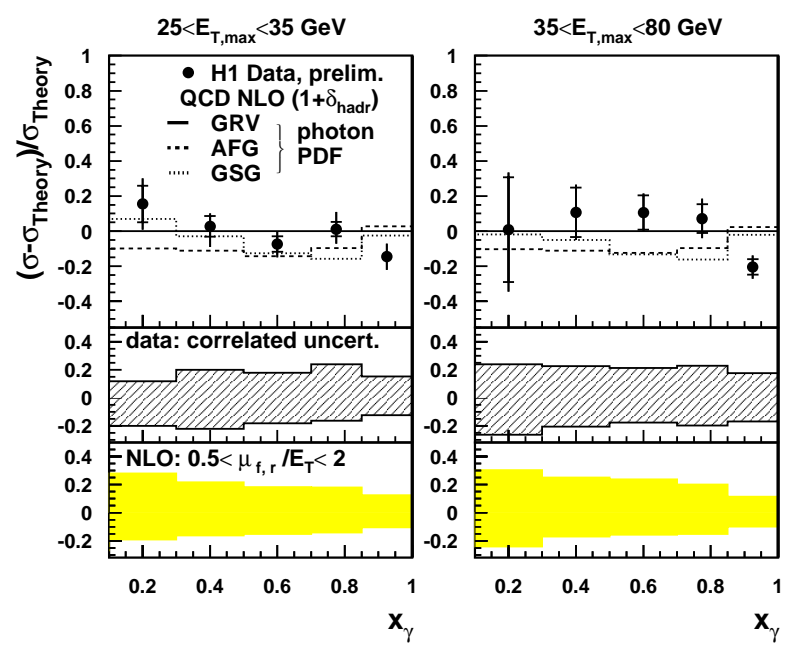

Figure 5: Relative difference of the $e p$ cross section $d \sigma / d x_{\gamma}$ for dijet production to the NLO QCD prediction. GRV-HO photon pdf's (dashed line) and to NLO calculations including corrections for hadronization effects (full line). The NLO scale uncertainties (shown as grey band) have a significant influence. For $x_{\gamma}<0.85$ 
the data is found to be well described by the prediction and for $x_{\gamma}>0.85$ to be slightly below the prediction.

This can also be inferred from fig. 5 which shows the relative difference between the data and the NLO calculations including hadronization corrections. In addition the data is compared to the NLO calculations using AFG-HO and GS-HO photon pdfs. The difference between different photon pdf's is less important, than the experimental and theoretical uncertainties. There is a noticeable difference between the $\mathrm{H} 1$ and

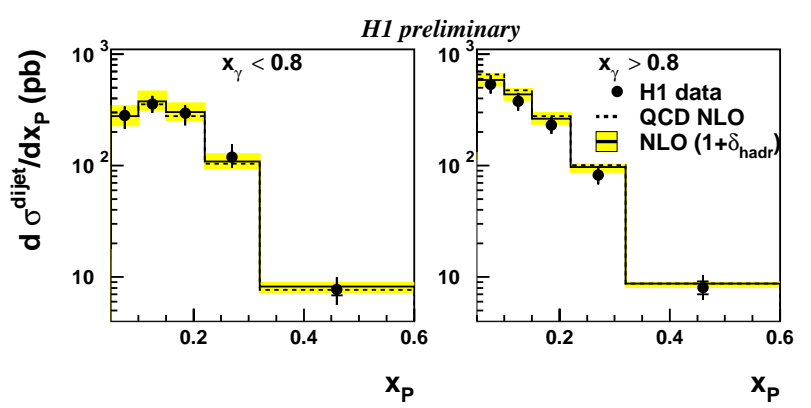

Figure 6: Differential $e p$ cross section $d \sigma / d x_{P}$ for dijet production.

ZEUS dijet results. A detailed comparison or re-analysis will probably be needed to clear this up.

The $\mathrm{H} 1$ collaboration has also measured the dijet cross section $d \sigma / d x_{P}$ as a function of $x_{P}$ for two different regions in $x_{\gamma}$ as shown in fig. 6 [i]. Again the data is compared to NLO QCD calculations with a GRV-HO photon and a CTEQ5M proton pdf and including hadronization corrections. Even at the highest $x_{P}$ the measured cross section agrees well with the QCD predictions. Different proton pdf sets (MRST99 1-3,CTEQ5M) result in differences of less than 5 percent for the predicted cross section for $x_{P}<0.1$ and up to 15 percent for $x_{P}>0.1$, which is of the same order as the scale uncertainties. In this part of the phase space, about $40 \%$ of the cross section is induced by gluons in the poorly constrained gluon distribution in the proton.

Recent measurements made by ZEUS test QCD predictions for the transition from quasi-real to virtual photons [8.

Fig. 7 shows the ratio $R=\sigma\left(x_{\gamma}<0.75\right) / \sigma\left(x_{\gamma}>0.75\right)$ as a function of $Q^{2}$ for different ranges of the mean transverse $E_{T}^{2}$ of the two jets. The fall of $R$ in the data with increasing $Q^{2}$ can be interpreted as a suppression of the virtual photon structure. HERWIG including a SAS1d photon pdf predicts this suppression, but underestimates the measured ratio $R$.

To summarize, we find that many recent measurements of jet production in photonproton scattering are described by NLO QCD or HERWIG with current pdfs of the photon and proton. Further investigations are needed to clarify disagreements and to reduce the experimental and the sometimes dominating theoretical uncertainties. However, this data will be useful in constraining parton densities in the future.

\section{References}

[1] S. Frixione and G. Ridolfi, Nucl. Phys. B507 (1997) 315.

M. Klasen, G. Kramer, Z. Phys. C 76 (1997) 67.

B.W. Harris, J.F. Owen, Phys. Rev. D 56 (1997) 4007.

P. Aurenche et al., Eur. Phys. J. C 17 (2000) 413. 


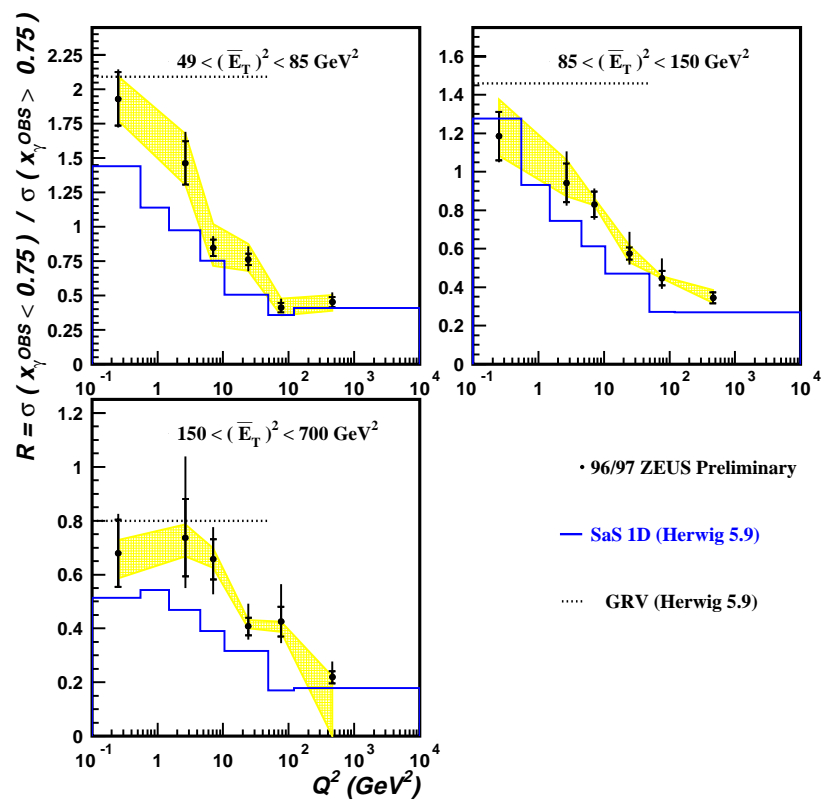

Figure 7: The ratio of cross sections $R$ as a function of $Q^{2}$.

[2] S.D. Ellis and D.E. Soper, Phys. Rev. D48 (1993) 3160.

[3] S. Catani, Yu.L. Dokshitzer, M.H. Seymour and B.R. Webber, Nucl. Phys. B 406 (1993) 187.

[4] H1 Collab., Measurement of single inclusive high $E_{T}$ jet cross-sections in photoproduction at HERA, Paper 813 to EPS HEP 2001.

[5] ZEUS Collab., Inclusive jet photoproduction at HERA, Prepared for ICHEP 98, Vancouver, (1998).

[6] ZEUS Collab., The structure of the photon and the dynamics of resolved photon processes in dijet photoproduction at HERA, Paper 1066 to ICHEP 2000, Osaka.

[7] H1 Collab. , Measurement of Dijet Cross Sections in Photoproduction at HERA, Paper 798 to EPS HEP 2001.

[8] ZEUS Collab., The $Q^{2}$ and $E_{T}^{2}$ dependence of dijet cross sections in gamma* $-p$ interactions at HERA, Paper 636 to EPS HEP 2001. 